Check for updates

Cite this: Chem. Commun., 2018, 54, 1121

Received 6th October 2017,

Accepted 17th November 2017

DOI: $10.1039 / c 7 c c 07748 c$

rsc.li/chemcomm

\section{Solvated-electron production using cyanocuprates is compatible with the UV-environment on a Hadean-Archaean Earth $\uparrow$ :}

\author{
Zoe R. Todd, (D) *ab Albert C. Fahrenbach, ${ }^{\text {bc }}$ Christopher J. Magnani, (D) ab \\ Sukrit Ranjan, (D) a Anders Björkbom, (D) ${ }^{\text {bd }}$ Jack W. Szostak (D) ${ }^{\text {bc }}$ and \\ Dimitar D. Sasselov ${ }^{a}$
}

\begin{abstract}
UV-driven photoredox processing of cyanocuprates can generate simple sugars necessary for prebiotic synthesis. We investigate the wavelength dependence of this process from 215 to $295 \mathrm{~nm}$ and generally observe faster rates at shorter wavelengths. The most efficient wavelengths are accessible to a range of potential prebiotic atmospheres, supporting the potential role of cyanocuprate photochemistry in prebiotic synthesis on the early Earth.
\end{abstract}

Many prebiotic syntheses of simple biomolecules, including ribonucleotides, amino acids, and lipid precursors, ${ }^{1}$ require simple 2- and 3-carbon sugar feedstock molecules, glycolaldehyde and glyceraldehyde. Past suggestions for the synthesis of simple sugars include the formose reaction, ${ }^{2}$ atmospheric photochemical production and subsequent transport to the surface, ${ }^{3}$ and delivery from space. ${ }^{4-6}$ However, unspecific products ${ }^{2}$ and large threshold concentrations $^{7-9}$ (formose reaction), and low yields ${ }^{3}$ (atmospheric production) are all drawbacks. Alternatively, Ritson and Sutherland $(2012)^{10}$ demonstrated that hydrogen cyanide can be converted to the glycolaldehyde and glyceraldehyde oxazolidinone derivatives via a Kiliani-Fischer homologation mechanism using cyanocuprate photoredox chemistry. This mechanism uses UV light to photooxidize the cyanocuprates, producing aqueous (solvated) electrons, which are capable of reducing HCN and 2-hydroxynitriles to imines, which can then hydrolyze to give rise to formaldehyde and $\alpha$-hydroxy aldehydes in a stepwise fashion.

Glyceraldehyde is the simplest sugar that contains a chiral center and can thus influence the stereochemistry in downstream synthesis. Glycolaldehyde and formaldehyde can form enantiomerically

\footnotetext{
${ }^{a}$ Department of Astronomy, Harvard-Smithsonian Center for Astrophysics, 60 Garden Street, Cambridge, MA 02138, USA. E-mail: zoe.todd@cfa.harvard.edu

${ }^{b}$ Howard Hughes Medical Institute, Department of Molecular Biology and Center for Computational and Integrative Biology, Massachusetts General Hospital, 185 Cambridge Street, Boston, MA 02114, USA

${ }^{c}$ Earth-Life Science Institute, Tokyo Institute of Technology, 2-12-1-IE-1 Ookayama, Meguro-ku, Tokyo, 152-8550, Japan

${ }^{d}$ Department of Biosciences, Åbo Akademi University, Åbo FI-20520, Finland

$\dagger$ In memory of Dr Alexei Trifonov.

\$ Electronic supplementary information (ESI) available. See DOI: 10.1039/c7cc07748c
}

enriched glyceraldehyde under chiral amino acid catalysts. ${ }^{11-13}$ Similarly, stereoselective tetrose $\mathrm{e}^{14,15}$ and pentose ${ }^{16}$ sugars were demonstrated under chiral amino acid catalysts and plausible prebiotic conditions. Steer et al. (2017) ${ }^{17}$ showed the selective synthesis of 2-deoxy-D-ribose from glycolaldehyde and formaldehyde under the influence of proteinogenic amino esters and amino nitriles. Plausible syntheses of glycolaldehyde and glyceraldehyde may thus be intimately connected with the issue of homochirality.

In the Ritson and Sutherland system, UV light photooxidizes the cyanocuprates, producing aqueous electrons, which are key for putting into motion the reaction network capable of producing glycolaldehyde and glyceraldehyde. (See Fig. 5 of Ritson and Sutherland $2012^{10}$ for a detailed mechanism.) Additional work by Ritson and Sutherland (2013) ${ }^{18}$ modified this cycle to include hydrogen sulfide as the stoichiometric reductant and produced free sugars upon $254 \mathrm{~nm}$ irradiation. The UV-wavelength dependence of the system with the addition of sulfide may not necessarily be the same as for the cyanocuprate cycle alone. Further work ${ }^{1}$ expanded upon the cyanosulfidic chemistry in Ritson and Sutherland (2012) ${ }^{10}$ and found that sugars, amino acids, ribonucleotides, and lipid precursors can be generated under UV irradiation at $254 \mathrm{~nm}$. In this system, copper is not strictly necessary, but can increase the overall efficiency.

Cyanocuprate photochemistry has only been studied at limited wavelengths (254 nm ${ }^{10,18-20}$ and $266 \mathrm{~nm}^{21}$ ), which are poor approximations of the spectral radiance illuminating the surface of the prebiotic Earth. ${ }^{22}$ The young Sun is thought to have been on average about $20-30 \%$ dimmer than today, but with a larger fraction of its radiation in the UV range. ${ }^{23,24}$ The early atmosphere (typically considered to be 1 bar of $\mathrm{N}_{2}$ and $\mathrm{CO}_{2}{ }^{25}$ ) was anoxic, ${ }^{26,27}$ providing ample radiation at wavelengths $>200 \mathrm{~nm}$, which are not screened out by the likely prebiotic atmospheric constituents. ${ }^{28}$

UV light is a potentially important source of energy to drive prebiotic reactions. ${ }^{29-31}$ Since photochemical reactions are generally wavelength-dependent, ${ }^{32}$ it is necessary to study the suggested prebiotic photochemistry at multiple wavelengths and fluxes more relevant to the prebiotic Earth. The flux from our experimental apparatus is consistent with the flux expected 


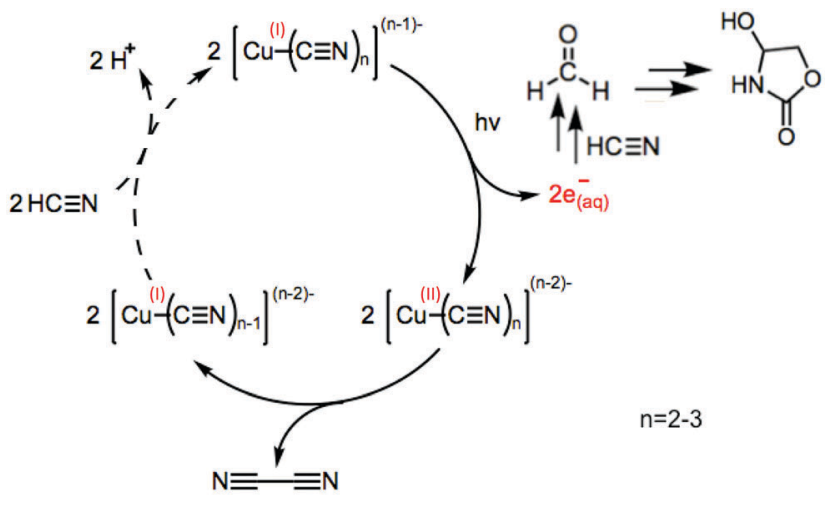

Net: $2 \mathrm{HC} \equiv \mathrm{N} \stackrel{\mathrm{Cu}(\mathrm{l}), \mathrm{hv}}{\longrightarrow} \mathrm{N} \equiv \mathrm{C}-\mathrm{C} \equiv \mathrm{N}+2 \mathrm{H}^{+}+2 \mathrm{e}_{(\mathrm{aq})}^{-}$

Scheme 1 The cyanocuprate photoredox process results in the production of solvated electrons, cyanogen, and protons and depletion of HCN. The reduction of $\mathrm{HCN}$ by solvated electrons yields formaldehyde after a few steps. This process is driven by UV-light, studied in the past at $254 \mathrm{~nm}$, and in this study, at a range of wavelengths from 215 to $295 \mathrm{~nm}$. The dashed lines indicate that some forms of the cyanocuprate complexes may not be replenished efficiently by $\mathrm{HCN}$ when $\mathrm{HCN}$ is limiting.

on the surface of the early Earth in the wavelength range of 235-255 nm to within an order of magnitude; our lamp provides roughly 6-9 times as much flux in this wavelength interval. Here, we investigate the wavelength dependence of the cyanocuprate photochemical process (Scheme 1) and assess if this reaction is plausible on the early Earth; other systems, such as the cyanosulfidic chemistry, will be addressed in future work. Studying the cyanocuprate photoprocess can act as a valuable test case for more complex chemical systems, such as those described in Ritson and Sutherland (2013) ${ }^{18}$ and Patel et al. (2015). ${ }^{1}$

We began by testing how the relative rates and quantum yields of the photoprocess depend on irradiation wavelengths from 215 to $295 \mathrm{~nm}$ (10 nm intervals, $10 \mathrm{~nm}$ bandwidths). These wavelengths of light (roughly 200-300 nm, or mid-range UV) were selected according to conditions expected on the surface of the early Earth. Aqueous solutions of dilute cyanocuprate complexes (63 $\mu \mathrm{M}$ copper(I) with three equivalents of cyanide) were prepared at neutral $\mathrm{pH}$ anaerobically. The absorption spectra of these solutions (Fig. 1A) display peaks at 210 and $234 \mathrm{~nm}$, indicating that the solution is primarily composed of dicyanocuprate complexes. The tricyanocuprate species has absorption maxima at $205 \mathrm{~nm}$ and $239 \mathrm{~nm}$. As the number of equivalents of cyanide per copper center is increased, the absorption spectrum changes from that characteristic of the dicyanocuprate to that of the tricyanocuprate (Fig. S5, ESI $\neq$ ). Irradiation of the solutions resulted over time in a decrease in the absorption intensity across the spectrum, the rate of which depended on the irradiation wavelength.

We attribute the decreases in absorbance to the following mechanism: photoexcitation by UV light of cyanocuprates releases solvated electrons, a fraction of which are scavenged by HCN to eventually yield sugar products. The cyanide ligands of the oxidized copper(II) cyanide complexes undergo reductive elimination, forming cyanogen and regenerating the copper(I) state. Each turn of the cyanocuprate photoprocess removes multiple HCN molecules per copper complex, causing the cyanocuprate distribution to favor complexes with lower coordination numbers and the observed decrease in absorbance. The relative rates of the reaction at different wavelengths were also monitored with a cyanide-selective electrode (Fig. S7, ESI $\ddagger$ ) to confirm the overall trend from the rates determined via absorbance measurements. LC-MS studies also confirmed the production of an oxazolidinone end product at all wavelengths tested (ESI, Scheme S1). This detection indicates that the production of simple sugars can occur even at more prebiotically relevant sub-millimolar concentrations of copper and cyanide, while previous experiments used higher initial concentrations $(200 \mathrm{mM}$ KCN and $10 \mathrm{mM} \mathrm{CuCN}){ }^{10}$

The decrease in absorbance of the cyanocuprate solution with irradiation time allows us to determine an apparent rate (Fig. 1B) for the overall photochemical process, which serves as a proxy for the relative rate of solvated electron production at each irradiation wavelength studied. In order to compare reaction rates at different irradiation wavelengths, we normalize the apparent
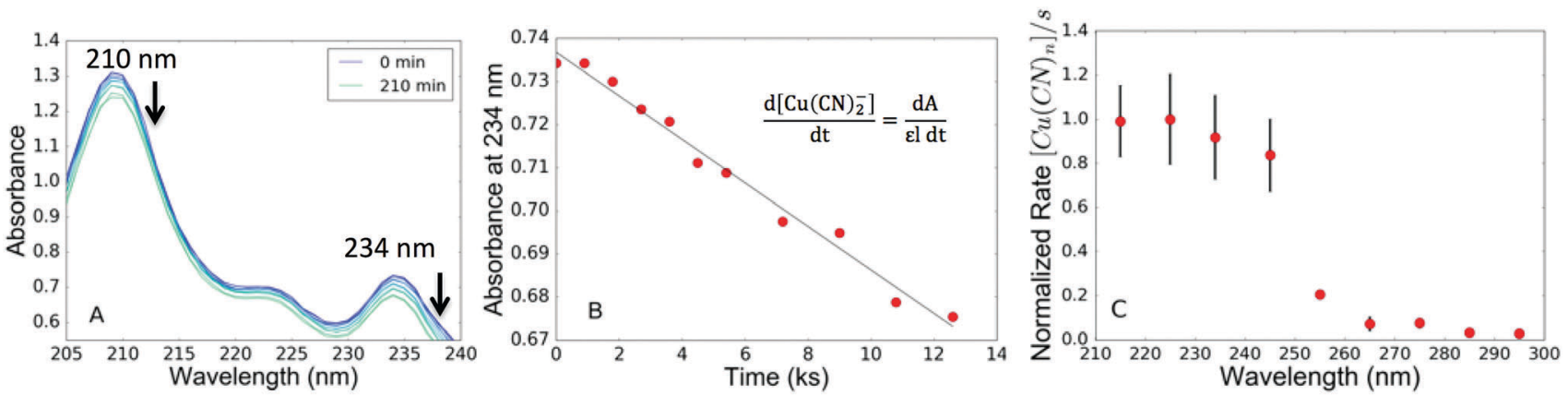

Fig. 1 (A) Absorption spectra of an aqueous solution of cyanocuprates ( $63 \mu \mathrm{M} \mathrm{CuCN}, 125 \mu \mathrm{M} \mathrm{KCN}$ ) during UV-irradiation. Arrows indicate the two absorption maxima at 210 and $234 \mathrm{~nm}$ decreasing as a function of irradiation time. (B) Absorbance at $234 \mathrm{~nm}$ as a function of irradiation time. The slope of the trendline measures the rate. (C) Action spectrum with the maximum value set to 1 . Each wavelength was tested in triplicate, with the values representing the averages and errors of the standard deviation. The rates are faster at wavelengths below roughly $250 \mathrm{~nm}$. The numerical values (and errors) for that data presented here are given in the ESI¥ (Section VII). 


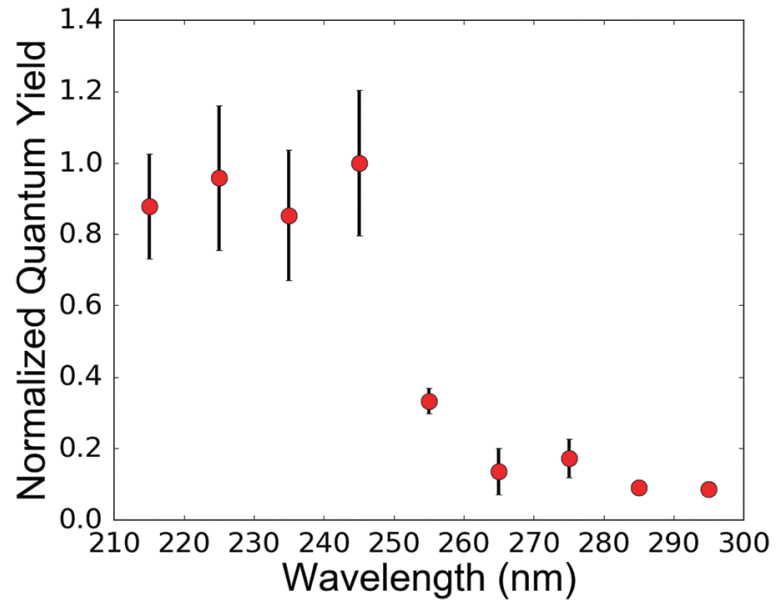

Fig. 2 Relative quantum yield (normalized such that the maximum is 1 ) as a function of irradiation wavelength. The quantum yield is the highest at a wavelength of $245 \mathrm{~nm}$. The numerical values (and errors) for the data presented here are given in the ESI (Section VII). Wavelength-dependent experiments employed solutions of $63 \mu \mathrm{M} \mathrm{CuCN}$ and $125 \mu \mathrm{M} \mathrm{KCN}$ prepared from concentrated stocks. The $\mathrm{pH}$ value was adjusted to 7.4 using $\mathrm{HCl}$. All preparations were done anaerobically, inside a glove box filled with an inert gas mixture $\left(98 \% \mathrm{~N}_{2}\right.$ and $\left.2 \% \mathrm{H}_{2}\right)$. The final samples were placed inside gas-tight screw-top Spectrosil cuvettes (Starna cells part number 9-Q-10-GL14-C) to ensure anaerobic conditions. For each experiment, $0.7 \mathrm{~mL}$ of cyanocuprate solution was irradiated. Every 15 minutes for the first two hours, and every 30 minutes thereafter (210 minutes total), UV-Vis absorption spectra were recorded in order to monitor the reaction and determine the rate.

rates by incident photon fluxes, to give the action spectrum ${ }^{34}$ (Fig. 1C; see the ESI for details). The normalized rate is larger at irradiation wavelengths $<250 \mathrm{~nm}$.

We calculated the relative quantum yields (number of reactions per absorbed photon) at the various irradiation wavelengths tested and normalized all quantum yields such that the maximum yield (at $245 \mathrm{~nm}$ irradiation, near the absorption maxima of the cyanocuprates) was set to 1 (Fig. 2). Two different mechanisms have been proposed for this process: (1) the Ritson-Sutherland mechanism ${ }^{10}$ postulates a photoionization as the underlying photoprocess, suggesting that any photon with energy greater than the activation energy of the process should be sufficient, and (2) Banerjee et al. $2014^{33}$ use theoretical calculations of electronic structures and a mechanism involving dipole-bound forms of $\mathrm{HCN}$ to predict a Gaussian efficiency centered at $265 \mathrm{~nm}$. Our results do not support this mechanism, given that the relative quantum yield at $265 \mathrm{~nm}$ is roughly a tenth of the maximum value at $245 \mathrm{~nm}$.

We next studied how the wavelength-dependent reaction rates compare to the spectral irradiation on the modern and prebiotic Earth. The relative rates determined herein suggest that the process proceeds more efficiently at wavelengths below $250 \mathrm{~nm}$, but shorter wavelengths are less accessible on the surface of a planet due to shielding by atmospheric gases and decreased solar output. We compute the intensity of light reaching the surface of the Earth as a function of wavelength by convolving the solar flux with the spectral screening by atmospheric gases (ESI, $\ddagger$ Section VI). We compute the relative rate of solvated-electron production as a function of wavelength by multiplying the action spectrum and integrated surface
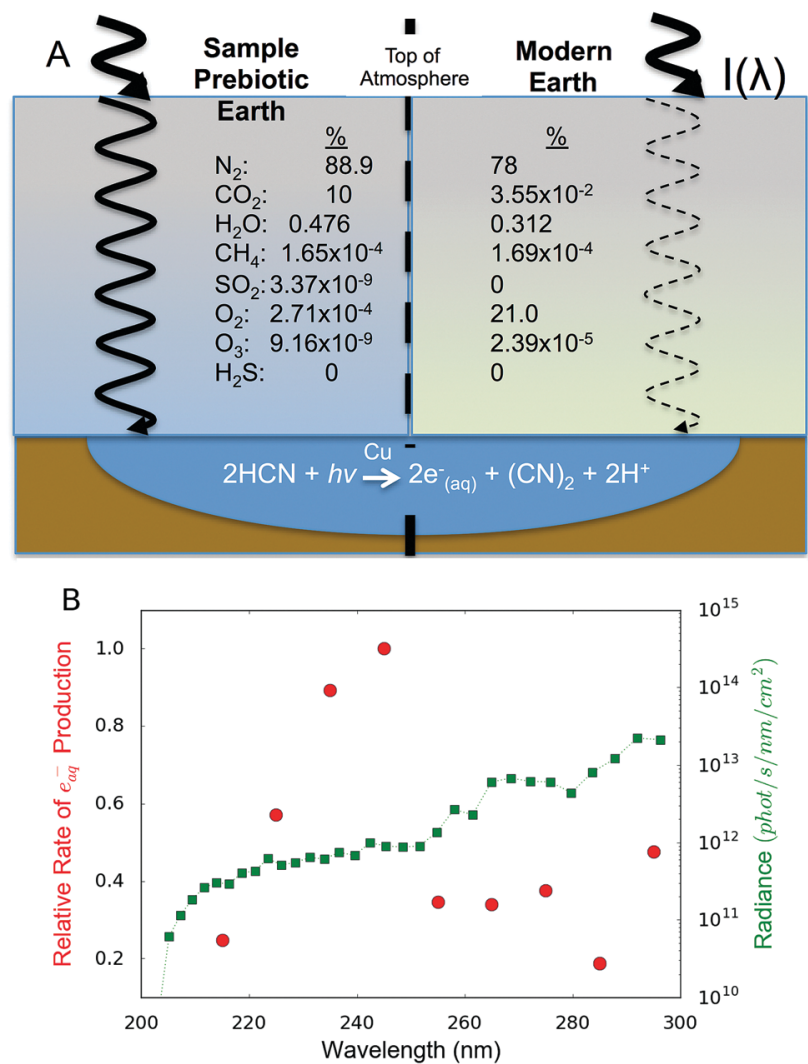

Fig. 3 (A) Schematic of the UV light reaching the surface of the Earth for a proposed prebiotic (left) and modern atmosphere (right), both with 1 bar and atmospheric compositions as noted. (B) The relative rate of solvatedelectron production occurring from the cyanocuprate photoprocess as a function of wavelength for a hypothetical prebiotic atmosphere (red circles; normalized so that the maximum is 1); for the modern Earth the rate is essentially zero and not shown. The highest relative rate occurs at $245 \mathrm{~nm}$. The Earth surface radiance (green squares) is shown for the early atmosphere. The UV wavelengths necessary for driving the cyanocuprate photochemical production of solvated electrons should be accessible on the early Earth and under a variety of atmospheres.

radiance at each wavelength. ${ }^{34}$ The resulting curve is shown in Fig. 3B (red dots); the area under it is commonly referred to as the relative dose rate. ${ }^{22}$

The modern surface of the Earth receives low levels of UV light (due to UV-shielding $\mathrm{O}_{2}$ and $\mathrm{O}_{3}$ ) leading to a UV flux inadequate to drive this photochemistry. Even when including the effects of the atmospheric attenuation and stellar spectral slope, we find that the most productive wavelength of irradiation remains $245 \mathrm{~nm}$ for the early Earth. Energy at the relevant wavelengths should be accessible on the early Earth for a range of plausible atmospheres, suggesting that this mechanism for simple sugar generation is viable. The peak wavelength does not precisely correspond with the $254 \mathrm{~nm}$ light that is typically used in prebiotic experiments, exemplifying the importance of wavelength dependence considerations. Our study, though carried out at specific individual wavelengths that do not mimic the spectrum of the Sun, when combined with information about the spectral flux on the early Earth, can provide insight into the plausibility of the process in the overall context of the UV environment on the early Earth. 
In summary, we performed a wavelength-dependent analysis to assess the plausibility of the synthesis of 2- and 3-carbon sugar building blocks (necessary for simple biomolecule synthesis) from photoredox processing of cyanocuprates. Past studies of the cyanocuprate photoprocess used monochromatic emission at $254 \mathrm{~nm}$, which is a poor proxy of the surface irradiation on the early Earth. In order to assess the prebiotic plausibility of this pathway, we measured the wavelength dependence and found that the process is more efficient at wavelengths $<250 \mathrm{~nm}$. The wavelength with the highest quantum yield ( $245 \mathrm{~nm})$ should be accessible not only in the atmosphere we tested here, but also in other plausible early Earth atmospheres. Thus, we argue that the photochemical cycling of cyanocuprates is a prebiotically plausible source of 2- and 3-carbon sugar building blocks on the early Earth, across a diversity of possible atmospheric states. This process should also work on early Mars (unless high levels of dust are present ${ }^{25,28}$ ) or exoplanets analogous to the young Earth orbiting stars with UV emission similar to the Sun.

We thank C. P. Tam and F. Ng for experimental and laboratory assistance. We thank R. Szabla and D. Bucher for helpful comments and discussions. We thank S. Trauger and others at the Harvard Small Molecule Mass Spectrometry Facility. This work was supported in part by grants from the Simons Foundation (290363 to J. W. S. and 290360 to D. D. S.) and we acknowledge the Harvard Origins of Life Initiative. J. W. S. is an investigator of the Howard Hughes Medical Institute. A. B. was supported by a grant from the Academy of Finland. A. B. is currently at Statens Serum Institut, Artillerivej 5, DK-2300 Copenhagen S, Denmark. C. J. M. is currently at the School of Medicine, Stanford University, 291 Campus Drive, Palo Alto CA, 94305.

\section{Conflicts of interest}

There are no conflicts to declare.

\section{Notes and references}

1 B. H. Patel, C. Percivalle, D. J. Ritson, C. D. Duffy and J. D. Sutherland, Nat. Chem., 2015, 7, 301.

2 A. Butlerov, Liebigs Ann. Chem., 1861, 120, 295.
3 C. E. Harman, J. F. Kasting and E. T. Wolf, Origins Life Evol. Biospheres, 2013, 43, 77.

4 C. F. Chyba, C. Sagan, L. Brookshaw and P. J. Thomas, Origins Life Evol. Biospheres, 1989, 19, 467.

5 C. F. Chyba, P. J. Thomas, L. Brookshaw and C. Sagan, Science, 1990, 249, 366.

6 M. Maurette, J. Duprat, C. Engrand, M. Gounelle, G. Kurat, G. Matrajt and A. Toppani, Planet. Space Sci., 2000, 48, 1117.

7 C. Reid and L. Orgel, Nature, 1967, 216, 455.

8 A. W. Schwartz and R. M. De Graaf, J. Mol. Evol., 1993, 36, 101.

9 N. Gabel and C. Ponnamperuma, Nature, 1967, 216, 453.

10 D. Ritson and J. D. Sutherland, Nat. Chem., 2012, 4, 895.

11 R. Breslow, V. Ramalingam and C. Appayee, Origins Life Evol. Biospheres, 2013, 43, 323.

12 R. Breslow and Z.-L. Cheng, Proc. Natl. Acad. Sci. U. S. A., 2010, 107, 5723.

13 J. E. Hein, E. Tse and D. G. Blackmond, Nat. Chem., 2011, 3, 704.

14 S. Pizzarello and A. L. Weber, Science, 2004, 303, 1151.

15 A. L. Weber and S. Pizzarello, Proc. Natl. Acad. Sci. U. S. A., 2006, 103, 12713.

16 S. Pizzarello and A. L. Weber, Origins Life Evol. Biospheres, 2010, 40, 3.

17 A. M. Steer, N. Bia, D. K. Smith and P. A. Clarke, Chem. Commun., 2017, 53, 10362.

18 D. J. Ritson and J. D. Sutherland, Angew. Chem., Int. Ed., 2013, 52, 5845.

19 A. Horvath, S. Papp and Z. Decsy, J. Photochem., 1984, 24, 331.

20 A. Horvath, Z. Zsilak and S. Papp, J. Photochem. Photobiol., A, 1989, 50, 129.

21 K. L. Stevenson and J. H. Jarboe, J. Photochem. Photobiol., A, 2002, 150, 49.

22 S. Ranjan and D. D. Sasselov, Astrobiology, 2016, 16, 68.

23 I. Ribas, G. F. Porto de Mello, L. D. Ferreira, E. Hebrard, F. Selsis, S. Catalan, A. Garces, J. D. do Nascimento Jr. and J. R. de Medeiros, Astrophys. J., 2010, 714, 384.

24 M. W. Claire, J. Sheets, M. Cohen, I. Ribas, V. S. Meadows and D. C. Catling, Astrophys. J., 2012, 757, 95.

25 S. Rugheimer, A. Segura, L. Kaltenegger and D. Sasselov, Astrophys. J., 2015, 806, 137.

26 J. Farquhar, J. Savarino, S. Airieau and M. H. Thiemens, J. Geophys. Res., 2001, 106, 32829.

27 A. A. Pavlov and J. F. Kasting, Astrobiology, 2002, 2, 27.

28 S. Ranjan and D. D. Sasselov, Astrobiology, 2017, 17, 169.

29 C. Sagan and B. N. Khare, Science, 1971, 173, 417.

30 C. Chyba and C. Sagan, Nature, 1992, 355, 125.

31 O. Pestunova, A. Simonov, V. Snytnikov, V. Stoyanovsky and V. Parmon, Adv. Space Res., 2005, 36, 214.

32 T. Matsunaga, K. Hieda and O. Nikaido, Photochem. Photobiol., 1991, 54, 403.

33 A. Banerjee, G. Ganguly, R. Tripathi, N. N. Nair and A. Paul, Chem. - Eur. J., 2014, 20, 6348.

34 An action spectrum is the rate of a photochemical process as a function of wavelength, normalized by flux. The action-spectral weighted surface radiance is the product of the action spectrum (Fig. 1C) and the surface radiance at each wavelength. 